\title{
Maternity waiting homes as a cost-effective intervention in rural Liberia
}

\author{
Julie M. Buser ${ }^{1, *}$ | Michelle L. Munro-Kramer ${ }^{1}$ | Monica Carney ${ }^{2}$ | Alphonso Kofa ${ }^{3}$ | \\ G. Gorma Cole ${ }^{3}$ | Jody R. Lori ${ }^{1}$
}

${ }^{1}$ Department of Health Behavior and Biological Sciences, University of Michigan School of Nursing, Ann Arbor, MI, USA

${ }^{2}$ Department of Economics and Accounting, College of the Holy Cross, Worcester, MA, USA

${ }^{3}$ Ministry of Health, Bong County Health Team, Suakoko, Bong County, Liberia

\section{${ }^{*}$ Correspondence}

Julie M. Buser, Department of Health Behavior and Biological Sciences, University of Michigan School of Nursing, Ann Arbor, MI, USA.

Email: jbuser@umich.edu

Funding Information

US Agency for International Development

\begin{abstract}
Objective: To analyze the cost-effectiveness of maternity waiting homes (MWHs) in rural Liberia by examining the cost per life saved and economic effect of $\mathrm{MWHs}$ on maternal mortality.

Methods: A cost-effectiveness analysis was used to evaluate costs and economic effect of MWHs on maternal mortality in rural Liberia to guide future resource allocation. A secondary data analysis was performed based on a prior quasi-experimental cohort study of 10 rural primary healthcare facilities, five with a $\mathrm{MWH}$ and five without a MWH, that took place from October 30, 2010 to February 28, 2015.
\end{abstract}

Results: Calculations signified a low cost per year of life saved at MWHs in a rural district in Liberia. Total population-adjusted number of women's lives saved over 3 years was 6.25.

Conclusion: While initial costs were considerable, over a period of 10 or more years $\mathrm{MWHs}$ could be a cost-effective and affordable strategy to reduce maternal mortality rates in Liberia. Discussion of the scaling up of $\mathrm{MWH}$ interventions for improving maternal outcomes in Liberia and other low- and middle-income countries is justified. Findings can be used to advocate for policy changes to increase the apportionment of resources for building more MWHs in low resource settings.

\section{KEYWORDS}

Cost-effectiveness analysis; Developing countries; International health policy; Liberia; Maternal and child health; Maternal mortality

\section{1 | INTRODUCTION}

Liberia ranks in the top 10 countries for maternal mortality worldwide with a ratio of 1072 maternal deaths per 100000 live births. ${ }^{1}$ This figure from the 2013 Liberia Demographic Health Survey (LDHS) maternal mortality rate (MMR) is not significantly different from the 2007 LDHS MMR of 994 deaths per 100000 live births. ${ }^{1}$ The Sustainable Development Goals (SDGs) aim to reduce the global maternal mortality ratio to less than 70 per 100000 live births by $2030 .^{2}$ One way to increase access to skilled healthcare professionals and reduce maternal morbidity and mortality in Liberia and other low- and middle-income countries (LMICS) is through the use of a maternity waiting home $(\mathrm{MWH}){ }^{3}$

Maternity waiting homes are residential dwellings located near to health facilities where women stay to await delivery and receive postpartum services. ${ }^{4}$ The purpose of MWHs is to provide a setting where women can stay during the final weeks of their pregnancy near a basic emergency obstetric and neonatal care (BEmONC) facility. ${ }^{4}$

A Cochrane review ${ }^{5}$ found insufficient evidence on which to base recommendations for practice and limited insight among available literature into the potential benefit of $\mathrm{MWHs}$. Although some $\mathrm{MWHs}$ have shown evidence of their value by reducing MMRs, ${ }^{6,7}$ there is a 
dearth of information on their economic practicality. A scoping review of the scientific literature on MWHs and neonatal outcomes in $\mathrm{LMICs}^{8}$ identified only one study recommending the scaling up of $\mathrm{MWHs}$ based on their cost-effectiveness. ${ }^{9}$

There is a gap in the literature on the economic practicality of $\mathrm{MWH}$ as an intervention to reduce the maternal mortality ratio and meet the SDGs. Research into the cost-effectiveness analysis (CEA) of $\mathrm{MWHs}$ is needed to inform policy at the national and international level whether to allocate resources for building additional homes in Liberia and other low resource settings. The purpose of the present study was to analyze the cost-effectiveness of $\mathrm{MWH}$ in rural Liberia by examining the cost per life saved and economic effect of $\mathrm{MWH}$ on maternal mortality to inform future policy.

\section{2 | MATERIALS AND METHODS}

For this CEA, a secondary data analysis was performed based on a prior quasi-experimental cohort study of 10 rural primary healthcare facilities, five with a $\mathrm{MWH}$ (exposed intervention group) and five without a MWH (unexposed comparison group) that took place from October 30, 2010 to February 28, 2015. ${ }^{3}$ The cohort study was interrupted by the Ebola virus disease outbreak (EVD) in early 2014, ${ }^{10}$ and the present study therefore reported on data from March 2011 (after baseline data collection ended) through September 2013 (prior to the beginning of the EVD outbreak). The five clinics in the $\mathrm{MWH}$ group were matched based on similar characteristics including size, location, distance from a paved road, and population demographics to the five clinics in the non-MWH group. All 10 clinics provided standard services including BEmONC and referral services. The 10 communities were all located in north central Liberia in Bong County, and all communities were within a 2-hour car ride to one of the three hospitals in Bong County providing comprehensive emergency obstetric and newborn care (CEmONC). During the period of initial data collection, the county had very few ambulances with most transfers to a higher level of care undertaken by private vehicle or taxi. Approval was obtained from the Institutional Review Board of the University of Michigan, and the study was cleared by the Liberian Ministry of Health. Consent was not required as no human subjects were involved in this secondary analysis. Microsoft Excel program version 2013 was used to display and statistically manage data.

component of the community buy-in and engagement that was utilized throughout the parent study. ${ }^{3}$ Communities unwilling to pledge services and materials were not eligible for participation. There was some variation across $\mathrm{MWH}$ sites in building costs and donations, with building costs dependent on whether a new home was built or whether an existing structure was used. ${ }^{3}$ Four MWHs were built from existing structures that were left in various levels of disrepair following the civil war-hence the variation in cost. One home was a new construction. The newly constructed MWH was built with a US \$25000 private donation.

The MWHs were available without charge to all pregnant women, and access was neither dependent on referral nor on the distance between a woman's home and the rural primary health clinic. ${ }^{3}$ Every $\mathrm{MWH}$ had a minimum of eight beds with mosquito netting, outdoor toilet facilities, and an outdoor kitchen with cooking utensils along with a sheltered area for firewood. ${ }^{3}$ Data on maternal and neonatal health outcomes, transfers to a CEmONC, maternal mortality, and $\mathrm{MWH}$ use were collected on a monthly basis by the Liberian research team from the facility logbooks.

Cost-effectiveness analysis is a recognized tool for guiding resource allocation ${ }^{11}$ and allows evaluation of a health intervention by asking, "How much health benefit do we get for our money?". ${ }^{12}$ Costeffectiveness analysis refers to the evaluation of alternatives according to both their costs and their efforts with regard to producing some outcome. ${ }^{13}$ Costs can be used to evaluate whether scarce resources are being consumed efficiently or if there is a possibility to redistribute them in a way to improve population health. ${ }^{14} \mathrm{WHO}$ criteria on costeffectiveness propose an intervention is highly cost-effective if it prevents a year of life lost for less than the national gross domestic product (GDP) per capita, cost-effective if one to three times the GDP per capita, and not cost-effective if more than three times the GDP per capita. ${ }^{15}$

A CEA was performed to examine the cost per year of life saved by a $\mathrm{MWH}$. Aggregate cost-effectiveness calculations for a rural district in Liberia were computed. Estimates of the catchment population provided by the Liberian Ministry of Health were included in the calculations. Catchment population is the population of people served by a particular health clinic. Data on maternal deaths were obtained from official registers kept by the local Liberian Ministry of Health at each facility. The locations were matched to controls based on the fact that they had similar characteristics, eliminating the need to adjust for these characteristics. The population-adjusted total lives saved was calculated as:

\footnotetext{
Population- adjusted lives saved $=\sum_{\{i=1\}}^{5}$ (maternal deaths in non MWH catchment area for group $i$ )

$\times\left(\frac{\text { number of women of childbearing age in } \mathrm{MWH} \text { catchment area for group } i}{\text { number of women of childbearing age in non MWH catchment area for group i }}\right)$

- (maternal deaths in MWH catchment area for group $i)$
}

Prior to construction of the $\mathrm{MWHs}$, all the potential communities were asked to pledge to provide services and raw materials (making bricks, hauling sand and gravel, cutting wood) as well as provide food and cooked meals for the construction teams. ${ }^{3}$ This was seen as a where $i$ is a group of catchment areas matched on approximate size, location, distance from a paved road, and population demographics.

The total cost per year of maternal life saved $\left(C_{Y L S}\right)$ per identified time period of the study ( 3 years) was calculated using the formula: 
TABLE 1 Characteristics of MWHs and non-MWHs.

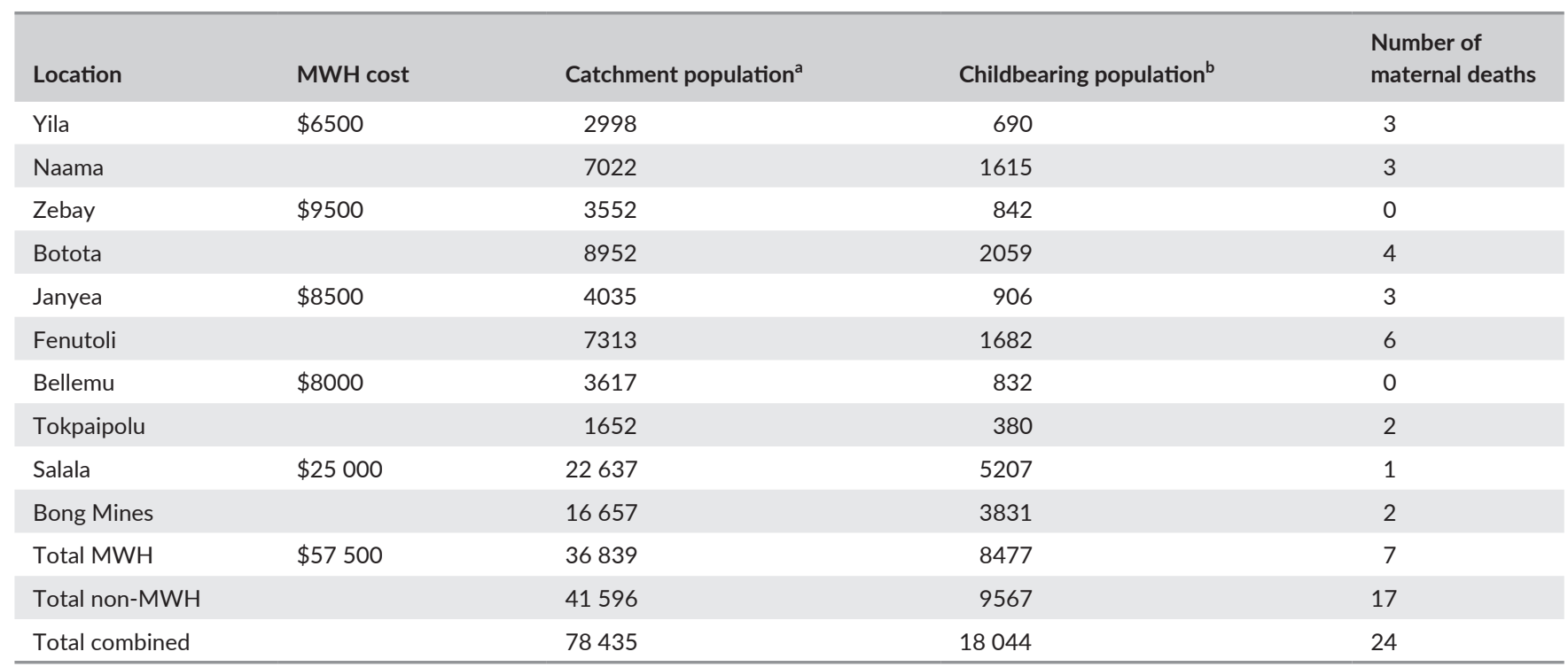

Abbreviation: $\mathrm{MWH}$, maternity waiting home.

${ }^{a}$ Data provided by the Ministry of Health, Bong County Health Team, 2012.

${ }^{\mathrm{b}} 23 \%$ of catchment community. ${ }^{20}$

$\left(C_{Y L S}\right)=\frac{\text { total discounted } C}{\left.\sum_{i=1}^{3} \text { (average total } L S / \text { year }\right) \times(2011 \text { value of average years of life remaining in year } i)}$.

Use of 2011 as the base year allows for comparison to the 2011 GDP in Liberia. Average years of life remaining was calculated as 34.7 years by subtracting the average age of maternal death in the collected data, 28.3 years, from the average life expectancy of 63 for women in Liberia. ${ }^{16}$ Since the given average life expectancy is unconditional on current age, the calculated average years of life remaining is likely a lower bound for the true value. To understand the increasing cost-effectiveness of $\mathrm{MWH}$ as a result of declining average fixed costs over time, cost per year of maternal life saved was extrapolated for longer periods of 5 and 10 years.

The overall costs of building all five of the MWHs was $\$ 57500$ (Table 1). Variable costs were also calculated for total labor and repairs each year, depending on the regularity with which the costs were incurred. Variable costs included wages, cooking utensils, plastic buckets, plastic mattresses, paint, roofing, and door replacement costs (Table 2).

Costs and benefits were discounted to account for the fact that costs and benefits in the future are worth less than if they were realized in the present. The widely accepted annual discount rate of $3 \%$ was used for such calculations. ${ }^{15}$ Specifically, variable costs incurred in the future were discounted, as was the value of future years of life remaining in the lives of women who were saved.

A final calculation of the total cost per year of life saved takes into account the decreased probability of death for the infant to whom the mother gave birth at the time of her death. Several sources in other African countries suggest the percentage point increase in the probability of an infant dying conditional on maternal death ranges from approximately $40 \%$ to $70 \%{ }^{17,18}$ Therefore, the total lives saved, inclusive of a lower bound value on the number of infants likely saved, was calculated as:

Total lives saved, mother or infant $=$ (number of maternal deaths from non-MWH catchment areas - number of maternal deaths from MWH catchment areas) $\times 1.4$.

Years of life saved for the infants took into account the full average of 63 years of life remaining for an infant. Stata version 14.2 software (StataCorp, College Station, TX, USA) and Student $t$ test were used to calculate the difference in lives saved between matched communities with and without a MWH. $P<0.05$ was considered statistically significant.

\section{RESULTS}

Results indicated a low cost per year of life saved at MWHs in a rural district in Liberia. A total of seven deaths in communities

TABLE 2 Variable costs of operating a MWH.

\begin{tabular}{lcl}
\hline Cost type & Cost amount & Frequency \\
\hline Wages & $\$ 840.00$ & Annual \\
\hline Cooking utensils & $\$ 270.00$ & Biennial \\
\hline Plastic buckets & $\$ 70.00$ & Biennial \\
\hline Plastic mattress & $\$ 240.00$ & Biennial \\
\hline Paint & $\$ 704.00$ & Biennial \\
\hline Roofing & $\$ 70.00$ & Triennial \\
\hline Doors & $\$ 170.00$ & Biennial \\
\hline
\end{tabular}


TABLE 3 Total number and cost per women's lives saved.

\begin{tabular}{|c|c|c|c|}
\hline & $3 y$ & 5 y (projected) & 10 y (projected) \\
\hline Total number of lives saved, women and infants (population-adjusted) ${ }^{b}$ & 11.25 & 18.7 & 37.4 \\
\hline Total cost per year of women's life saved (USD, discounted) d,e $^{\mathrm{d}}$ & $\$ 574$ & $\$ 416$ & $\$ 309$ \\
\hline
\end{tabular}

${ }^{\text {a }}$ Population-adjusted lives saved account for differences in population between $\mathrm{MWH}$ and non-MWH communities as a factor explaining differences in maternal death numbers within a community.

${ }^{\text {b}}$ Years of infants' lives saved is not based on collected data, but rather estimated based on the likelihood of the infant's survival after a maternal death calculated in other studies.

${ }^{\complement}$ Variable costs include wages, cooking utensils, plastic buckets, plastic mattresses, paint, roofing, and door replacement costs as given in Table 2.

${ }^{\mathrm{d}}$ Total cost of year of life saved calculated using population-adjusted lives saved.

${ }^{e}$ Future years of life are discounted annually using a rate of 3\% using 2011 as the base year. Variable costs incurred after 2011 are similarly discounted in total cost per year of life saved calculations.

${ }^{\mathrm{f}}$ Assumption made that there is an $80 \%$ chance of the infant dying as a result of maternal death.

with a $\mathrm{MWH}$, compared to 17 deaths in communities without a $\mathrm{MWH}$, were reported during the study (Table 1). The total number of women's lives saved over 3 years was 10 (17 maternal deaths non-MWH - 7 maternal deaths $\mathrm{MWH}$ ), or 6.25 when taking into account population differences between treatment communities and matched control communities (Table 3). The difference in lives saved between matched communities with and without a $\mathrm{MWH}$ (10) revealed a significant difference with a $P$ value of 0.047 . The number of births in communities with MWHs was 248 and in communities without MWHs, the number of births was 255 . When extrapolated over 10 years, the total number of women's lives saved (population-adjusted) would be:

$$
\frac{6.25 \text { lives saved }}{3 \text { year study period }} \times 10 \text { years }=20.8 \text {. }
$$

The total cost per year of maternal life saved (discounted) over 3 years was calculated as (Table 3 ): half of our point estimate, then the intervention would be cost-effective at exactly 3 years. Additional analyses included the likely secondary impact of neonatal deaths, as well as discounting of costs and benefits to account for the lessened value today of future years of life and/or repair and upkeep costs. For the discounted total cost of the MWHs, the fixed cost of building the $5 \mathrm{MWHs}$ was added to the discounted variable costs for all $5 \mathrm{MWH}$ s over each of the time periods (Table 3). The discounted variable costs were based on estimates of supply, repair, and wages costs. When the likelihood of an infant death was included in the discounted total cost per year of maternal life saved, the cost per year of maternal life saved was just $\$ 381$ over the 3-year study period (Table 3).

\section{4 | DISCUSSION}

The secondary data analysis in the present study investigated the costeffectiveness of $\mathrm{MWH}$ in rural Liberia along with the economic effect

$$
\frac{\text { total discounted } C}{\sum_{i=1}^{3}(\text { average total LS/year }) \times(2011 \text { value of average years of life remaining in year } i)}=
$$

Using the same methodology, cost per year of maternal life saved over 10 years would be $\$ 309$. The GDP per capita in Liberia was $\$ 379.69$ in 2011, the first year of the study and base year for all discounted calculations. ${ }^{16}$ Therefore, the discounted total cost per year of maternal life saved was highly cost-effective at $\$ 309$. The MWHs would become cost-effective by 2 years and highly cost-effective by 7 years. If all the MWHs had a building cost of $\$ 6500$, as a lower bound, they would be cost-effective within 1 year, while if they all had a building cost of $\$ 25000$, an upper bound, they would be cost-effective within 3 years. Even if only 1.05 lives were saved each year, or just over 12 per 100000 community members in the childbearing population, about of MWHs on maternal mortality. To our knowledge, this analysis was the first to provide insight into the cost-effectiveness of MWHs. Results demonstrated that MWHs were a highly cost-effective and affordable strategy to reduce maternal mortality in Liberia in a short time period.

Costs associated with the MWHs varied significantly across communities in the study. In most communities existing structures were renovated, while in one community a new home was constructed. Given the wide range in budget between old and new structures, the economic effectiveness estimates of aggregate costs could be skewed. In some communities, much of the construction material and services were donated thereby lowering costs. The study found 
additional variation between communities in the amount of raw materials and services donated that could reduce costs of a $\mathrm{MWH}$ compared to others.

The highest combined total of maternal deaths occurred in communities without a $\mathrm{MWH}$. The number of maternal lives saved was extrapolated over 5 and 10 years to demonstrate the long-term economic benefit of investing in the MWHs. The scaled-up estimates demonstrated that making a large one-time investment to build a $\mathrm{MWH}$ would have an enormous long-term benefit in increasing access to skilled obstetric care and reducing maternal mortality.

Cost-effectiveness results modeled over 3,5 , and 10 years illustrated that the cost per year of maternal life saved decreased over time as a result of falling average fixed costs, and that the cost per year of life saved fell as more lives were saved. Over 10 years, the cost per maternal life saved dropped to \$309. Using WHO costeffectiveness thresholds in relation to the Liberian GDP per capita, it was concluded that building $\mathrm{MWH}$ in rural Liberia would be a highly cost-effective intervention over 10 or more years and, if years of infant lives saved were included, possibly as soon as 5 years. When compared to the average GDP per capita in low income countries in 2011 of $\$ 694.94$, the MWHs would be highly cost-effective within 3 years, though costs would also likely be higher in these countries.

The secondary cost analysis study had several limitations. The prior cohort study ${ }^{3}$ did not collect data with a cost-effective analysis in mind therefore the amount of financial information to evaluate was limited. Additionally, the prior cohort study did not capture a number of characteristics that may have impacted differences including total number of births, total fertility rate, and maternal risk. A discussion of how these costs and characteristics influenced results would have been useful. Future studies should explore the role of various types of costs associated with MWHs. Another limitation of the current study was that the effect of MWHs on newborn health was incorporated into calculations based on effects found in studies in other African countries. Given the strong link between maternal and newborn health, the cost-effectiveness analysis would be strengthened by directly investigating with primary data collection the combined effect of $\mathrm{MWH}$ on both women and newborns. Despite these limitations, the present study was the first one to analyze the cost-effectiveness of $\mathrm{MWHs}$ in a low income country and to provide important insights into the economic effect of MWHs on maternal mortality.

With low average costs in the long term, MWHs could be a cost-effective and affordable strategy for reducing maternal mortality in Liberia. This economic analysis has added to the existing body of evidence that MWHs should be considered as a health system strengthening effort and a geographic bridge to maternal healthcare. ${ }^{6,7,19}$ Further work is needed to substantiate the cost-effectiveness of MWHs in other LMICs. The scarcity of rich experimental cost-related data associated with $\mathrm{MWH}$ needs to be addressed. Only by presenting solid information on the economic benefits of investing in MWHs will policy makers be able to make informed decisions.

\section{AUTHOR CONTRIBUTIONS}

JMB contributed to the conception and design of the study, the interpretation and analysis of the data, and to writing the manuscript. MMK and JRL contributed to the conception of the study, developed the protocol for the original study, had knowledge and ownership of the data, contributed to data collection, the interpretation and analysis of the data, and to revising the manuscript. MC contributed to the interpretation and analysis of the data and content expertise, and to revising the manuscript. AK and GGC contributed to data collection, analysis of the data and to revising the manuscript. All the authors reviewed and approved the final draft.

\section{ACKNOWLEDGMENTS}

The original study was supported by the US Agency for International Development, Child Survival Grant USAID-M-OOA-GH-HSR-10-40 (Jody R. Lori). The authors gratefully acknowledge Dr. Olga Yakusheva, Associate Professor, School of Nursing, School of Public Health, Institute for Healthcare Policy and Innovation, at the University of Michigan for her valuable support.

\section{CONFLICTS OF INTEREST}

The authors have no conflicts of interest.

\section{REFERENCES}

1. Demographic and Health Surveys Program. Liberia demographic and health survey. 2013. http://dhsprogram.com/what-we-do/survey/ survey-display-435.cfm. Accessed October 26, 2018.

2. United Nations. Sustainable Development Goal 3: Ensure healthy lives and promote well-being for all at all ages. 2017. https://susta inabledevelopment.un.org/sdg3. Accessed July 5, 2018.

3. Lori JR, Munro ML, Rominski S, et al. Maternity waiting homes and traditional midwives in rural Liberia. Int J Gynecol Obstet. 2013;123:114-118.

4. World Health Organization. Maternity waiting homes: a review of experiences. 1996. http://apps.who.int/iris/bitstream/10665/ 63432/1/WHO_RHT_MSM_96.21.pdf. Accessed July 5, 2018.

5. van Lonkhuijzen L, Stekelenburg J, van Roosmalen J. Maternity waiting facilities for improving maternal and neonatal outcome in low-resource countries. Cochrane Database Syst Rev. 2012;(10): CD006759.

6. Kelly J, Kohls E, Poovan P, et al. The role of a maternity waiting area (MWA) in reducing maternal mortality and stillbirths in high-risk women in rural Ethiopia. BJOG. 2010;117:1377-1383.

7. Lori JR, Wadsworth AC, Munro ML, Rominski S. Promoting access: The use of maternity waiting homes to achieve safe motherhood. Midwifery. 2013;29:1095-1102.

8. Buser JM, Lori JR. Newborn outcomes and maternity waiting homes in low and middle-income countries: A scoping review. Matern Child Health J. 2017;21:760-769.

9. Andemichael G, Haile B, Kosia A, Mufunda J. Maternity waiting homes: A panacea for maternal/neonatal conundrums in Eritrea. $J$ Eritrean Med Assoc. 2009;4:18-21.

10. Lori JR, Rominski SD, Perosky JE, et al. A case series study on the effect of Ebola on facility-based deliveries in rural Liberia. BMC Pregnancy Childbirth. 2015;15:254. 
11. Ha DA, Chisholm D. Cost-effectiveness analysis of interventions to prevent cardiovascular disease in Vietnam. Health Policy Plan. 2010;26:210-222.

12. Owens DK. Interpretation of cost-effectiveness analyses. J Gen Intern Med. 1998;13:716.

13. Levin HM, McEwan PJ. Cost-effectiveness analysis: Methods and applications. Thousand Oaks, CA: Sage; 2000.

14. Johns B, Baltussen R, Hutubessy R. Programme costs in the economic evaluation of health interventions. Cost Eff Resour Alloc. 2003;1:1.

15. Baltussen R, Adam T, Tan-Torres Edejer T, Hutubessy R, Acharya A. Methods for generalized cost-effectiveness analysis. Making choices in health: WHO guide to cost-effectiveness analysis. 2003. https:// www-who-int.proxy.lib.umich.edu/choice/publications/p_2003_ generalised_cea.pdf. Accessed April 18, 2019.

16. World Bank. GDP per capita (current US \$). 2011. https://data.world bank.org/indicator/NY.GDP.PCAP.CD. Accessed November 1, 2018.
17. Moucheraud C, Worku A, Molla M, Finlay JE, Leaning J, Yamin AE. Consequences of maternal mortality on infant and child survival: $A$ 25-year longitudinal analysis in Butajira Ethiopia (1987-2011). Reprod Health. 2015;12:S4.

18. Finlay JE, Moucheraud C, Goshev S, et al. The effects of maternal mortality on infant and child survival in rural Tanzania: A cohort study. Matern Child Health J. 2015;19:2393-2402.

19. Lee AC, Lawn JE, Cousens S, et al. Linking families and facilities for care at birth: What works to avert intrapartum-related deaths? Int $J$ Gynecol Obstet. 2009;107(Suppl.1):S65-S85, S86-8.

20. Liberia Institute of Statistics and Geo-Information Services (LISGIS). 2008 Population and Housing Census Final Results. 2009. https:// www.lisgis.net/pg_img/NPHC\%202008\%20Final\%20Report.pdf. Accessed July 13, 2018. 